\title{
Mixed self-assembled monolayers of azobenzene photoswitches with trifluoromethyl and cyano end-groups
}

\author{
Daniel Brete, ${ }^{1,2}$ Daniel Pryzembel ${ }^{1}$, Christian Eickhoff, ${ }^{1,2}$ \\ Robert Carley, ${ }^{1,2}$ Wolfgang Freyer, ${ }^{2}$ Karsten Reuter, ${ }^{3}$ Cornelius \\ Gahl, ${ }^{1}$ and Martin Weinelt ${ }^{1}$ \\ ${ }^{1}$ Freie Universität Berlin, Fachbereich Physik, Arnimallee 14, 14195 Berlin, Germany \\ ${ }^{2}$ Max-Born-Institut, Max-Born-Str. 2A, 12489 Berlin, Germany \\ ${ }^{3}$ Technische Universität München, Lichtenbergstrasse 4, 85748 Garching, Germany \\ E-mail: weinelt@physik.fu-berlin.de
}

\begin{abstract}
Mixed self-assembled monolayers (SAMs) of alkanethiolates carrying azobenzene chromophores with either a trifluoromethyl or a cyano substituent have been studied. High-resolution X-ray photoelectron spectroscopy proves that the ratio of adsorbed molecules can be arbitrarily adjusted via the molar fractions in solution. As a function of these molar fractions core-level-shifts are observed which are attributed to local work function changes. By simulating the electric dipole field-distribution the continous core-level-shifts are ascribed to a homogeneous mixture of molecules with different end groups adsorbed on adjacent lattice sites. Near-edge X-ray absorption fine structure measurements reveal formation of well-ordered SAMs. Despite the difference in dipole moment of the end groups, the molecular tilt and twist angles are identical for both single-component SAMs and a $1: 1$ mixed SAM.
\end{abstract}

Submitted to: J. Phys.: Condens. Matter

PACS numbers: 


\section{Introduction}

Self-assembled monolayers (SAMs) are frequently proposed as building blocks of supermolecular structures to functionalize surfaces $[1,2,3]$. In particular alkanethiols provide a strongly-bound anchor with physically flexible spacer, which can carry large end groups and thereby decouple functional moieties from the substrate [4]. Thiols chemisorb with the sulphur atom at coinage-metal surfaces with a close-to-upright molecular orientation [5, 6, 7]. The van der Waals interaction among the alkane spacers supports, in addition, lateral order in a densely-packed SAM. Particularly for biphenyl- and terphenyl-substituted alkanethiols, it is well-established that molecular tilt angles can be adjusted by combining the aliphatic linker with an aromatic end group $[8,9,10]$. To assemble switchable SAMs, azobenzene chromophores have been attached to aliphatic and aromatic linkers $[11,12,13,14,15,16,17,18,19]$.

The identical orientation of the molecular building blocks and the concomitant dense packing of SAMs are a mixed blessing. On the one hand they are necessary for well-ordered self-assembly from solution and guarantee robustness of the SAM in ambient conditions. On the other hand steric hindrance and excitonic coupling among the chromophores can strongly reduce the photoisomerization of azobenzene [11, 20]. To solve these problems several strategies have been pursued. Asymmetric azobenzene disulphides with a simple alkyl chain on the second sulphur binding-group have been used to dilute the surface coverage of the photoswitch in the $\operatorname{SAM}[11,18,21,22]$. When single molecules carrying azobenzene were adsorbed at defect sites of a pure alkanethiol SAM, photoswitching of these isolated entities was verified by scanning tunneling microscopy [16]. In principle this defect density can be enhanced by UV irradiation, leading to active sites where appropriate molecules can be deposited in an exchange reaction $[1,23]$. An alternative method to dilute the thiol species is to grow porous networks $[24,25]$ or use macromolecular platforms [26]. Bulky molecular tripods have also been successfully employed to anchor photoswitchable azobenzene to a metal surface [27]. Most recently reversible photoswitching has been demonstrated for conjugated azobenzenes in densely packed SAMs by measuring small work-function changes [19].

Besides these strategies it would be very desirable to mix molecules in solution and be able to form a matrix of functional photoswitches and intermolecular spacers by self-assembly at the surface [28]. So far co-adsorption has mainly been studied for simple alkanethiolates of various chain lengths [29]. Even if two molecules $A$ and $B$ are only slightly different, they usually become immiscible at the gold surface [1].

In most cases the attractive intermolecular van der Waals interactions of species $A$ and $B$ follow a hierarchy, e.g., $A A>B B>A B$. Therefore exchange reactions during the self-assembly process lead to phase separation, i.e. islands of $A$ and $B$, or uniform layers of $A$ are found after typical immersion times of 24 hours. Obviously, very similar molecules are needed to prepare mixed SAMs.

As a prototype system we have studied mixed SAMs of alkanethiolates carrying 

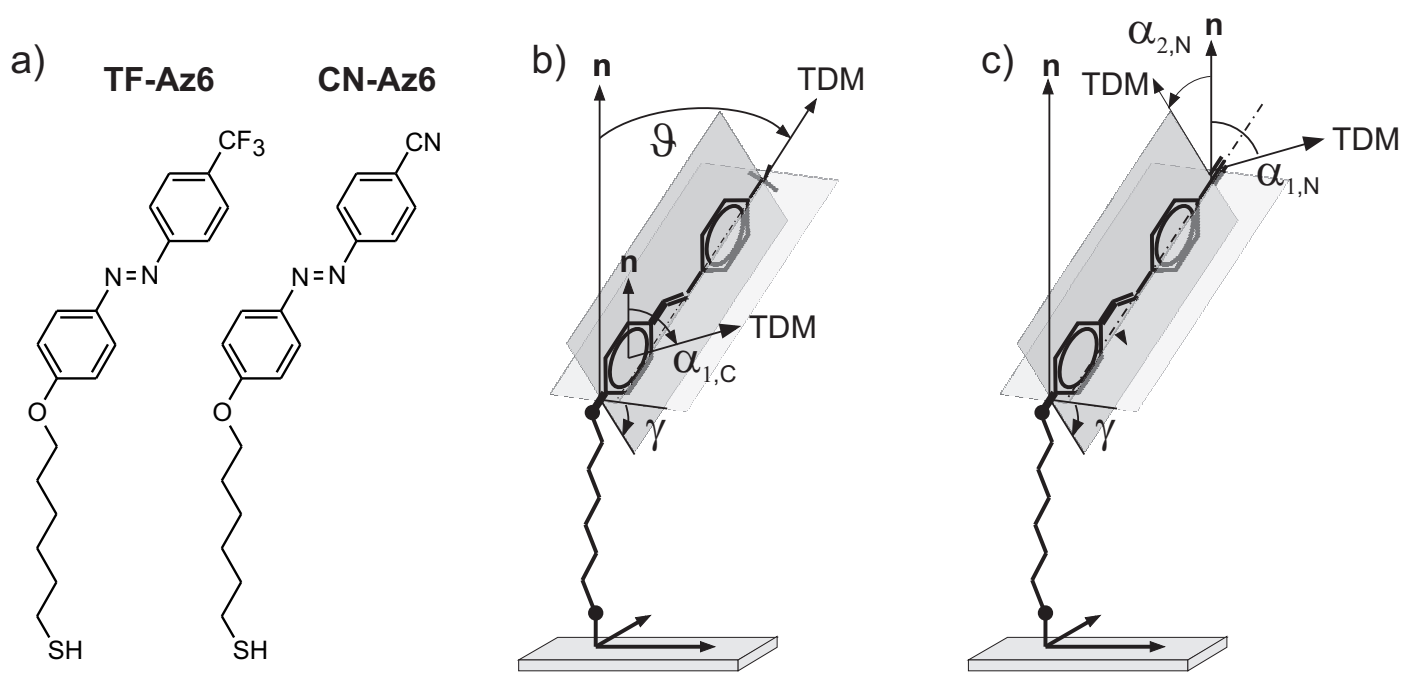

Figure 1. a) Structural formula of the two azobenzene alkanethiolates: The $\mathrm{CF}_{3^{-}}$ derivative TF-Az6 and the nitrile CN-Az6. b) Geometrical structure and transition dipole moments of TF-Az6. $\alpha_{1, C}$, and $\vartheta$ describe the mean tilt angles with respect to the surface normal, while $\gamma$ is the twist angle of the azobenzene chromophore. The orientation of the TDMs is probed in X-ray absorption at the C1s edge. The TDM of the $\mathrm{C} 1 s$ to $\pi^{*}$ transition is aligned parallel to the surface for $\gamma=90^{\circ}$. c) $\alpha_{1, N}, \alpha_{2, N}$ describe the mean tilt angles of the cyano group in CN-Az6. The orientation of the TDMs is probed in X-ray absorption at the N1s edge.

azobenzene chromophores with either a trifluoromethyl or a cyano end group attached in the para position (see Fig. 1). While exchange of the end group only slightly modifies the molecular structure, it alters the dipole moment of the molecules. Furthermore, end groups allow the optical absorption of the azobenzene chromophore to be tuned. In the present article we show that trifluoromethyl- and cyano-terminated azobenzene photoswitches form well-ordered mixtures of SAMs. The molecular orientation of the chromophores is deduced from near-edge X-ray absorption fine structure (NEXAFS) measurements. The molecular tilt and twist angles of $\sim 30^{\circ}$ and $\sim 60^{\circ}$ are identical for both single-component SAMs and a 1:1 mixture. High-resolution X-ray photoelectron spectroscopy (XPS) proves that the ratio of adsorbed molecules can be deliberately tuned by the ratio of their concentrations in solution. Adsorption of the trifluoromethylterminated molecules is favored by about a factor of two. The observed core-level shifts reflect the local work-function change, suggesting that the molecules form a homogenously mixed SAM.

\section{Experiment}

\subsection{Sample preparation}

6-(4-trifluoromethyl-4'-azobenzeneoxy)-hexane-1-thiol and 6-(4-cyano-4'-azobenzeneoxy)hexane-1-thiol were synthesized and purified as described in the online material and 
Ref. [30]. The purity of the compounds has been verified by ultrahigh-performance liquid chromatography. The structural formulas of the molecules, referred to as TF-Az6 and CN-Az6, are illustrated in Fig. 1. $300 \mathrm{~nm} \mathrm{Au} \mathrm{films} \mathrm{on} \mathrm{mica,} \mathrm{annealed} \mathrm{after} \mathrm{gold}$ deposition (Georg Albert, PVD coating), are used as substrates for SAM formation. The polycrystalline surface exhibits large $\mathrm{Au}(111)$ terraces of a few hundred nanometer width [31]. In the self-assembly process, contaminations like sulphur and carbon are displaced by the adsorbing molecules. The quality of each preparation was checked by recording S2 $p$ XP spectra. For SAM preparation the gold-covered mica substrates are immersed in $0.1 \mathrm{mM}$ ethanolic solution (total thiol concentration) for 24 hours at room temperature. These adsorption solutions were prepared from $1 \mathrm{mM}$ stock solutions of the two components. The concentration of the stock solutions was verified by photometry. In the binary mixtures the error of concentrations is estimated to $3 \%$ of the total chromophore concentration. In order to avoid weakly bound molecules residing on top of the SAM, all samples were thoroughly rinsed with pure ethanol after immersion and blown dry with argon. Thereafter samples were transported under argon and transferred into ultrahigh vacuum via a fast load-lock.

\subsection{Quantitative XPS}

For XPS measurements we used a newly built experimental apparatus equipped with a monochromatized Al $\mathrm{K}_{\alpha}$ source (Scienta MX650) and a Scienta SES200 hemispherical electron analyzer. The XPS apparatus has a resolution of $0.3 \mathrm{eV}$. All XPS measurements were performed at $300 \mathrm{~K}$. The spectra were recorded in grazing emission ( $65^{\circ}$ off-normal) to enhance surface sensitivity. The X-ray source has a line-focus elongated parallel to the entrance slit of the hemispherical electron analyzer. The large X-ray spot helps to reduce radiation damage to below the detection limit [32]. Au 4f peak positions and integrals were obtained by fitting a Voigt doublet and a Shirley type background. All spectra are normalised to the integral of the $\mathrm{Au} 4 \mathrm{f}$ doublet. The binding energy is referenced to the $\mathrm{Au} 4 \mathrm{f}_{7 / 2}$ level at $83.95 \mathrm{eV}$ of the same sample.

Peak integrals and positions for $\mathrm{F} 1, \mathrm{O} 1 s$ and the $\mathrm{CF}_{3}$-component of the $\mathrm{C} 1$ s spectra were obtained by fitting a single Gaussian and a linear background. Three Gaussians and a linear background were used for the $\mathrm{C} 1 \mathrm{~s}$ main peak to accommodate the aromatic carbon in the chromophore, the alkane chain and the chemically shifted $\mathrm{C}-\mathrm{O}$ and $\mathrm{C} \equiv \mathrm{N}$ components.

N1s integrals were obtained after subtracting a linear background. A fit of Eq. 3 to these integrals (Fig. 3a) yielded peak intensities subsequently used to fit the N1s spectra with two (one in the case of pure TF-Az6) Gaussians on a linear background to obtain the peak positions of the $\mathrm{N}=\mathrm{N}$ and the $\mathrm{C} \equiv \mathrm{N}$ component.

\subsection{NEXAFS}

NEXAFS spectroscopy was carried out at beamline UE52-SGM of the storage ring BESSY II (Helmholtz-Zentrum Berlin). The degree of polarization of the beamline is 
expected to be $96 \pm 2 \%$. Auger electrons were recorded in the direction perpendicular to the polarization of the incident X-ray beam after passing through a hemispherical electron analyzer with a five channel detector (Omicron EA125). To reduce radiation damage the sample was kept at liquid nitrogen temperature [33] and a fast shutter was used to block the beam during movement of the monochromator and undulator. The shutter is equipped with a GaAs photodiode, which records the X-ray flux alternatingly with the measurement and is used to normalize the NEXAFS spectrum. Further details are given in Ref. [20].

\section{Results and Discussion}

\subsection{SAM composition}

The chemical composition of the SAMs was studied by X-ray core-hole spectroscopy with monochromatized $\mathrm{Al} \mathrm{K} \alpha$ radiation $(h \nu=1486.7 \mathrm{eV})$. XP spectra were recorded for samples immersed in solutions of TF-Az6 or CN-Az6, as well as in mixtures with 0.10, $0.20,0.35,0.50,0.65,0.80$ and $0.90 \mathrm{TF}-\mathrm{Az} 6$ content. The quality of each preparation was verified by XPS of the thiol headgroup (not shown). All sulphur XP spectra consist of a single $2 p$ doublet with an intensity ratio of $2: 1$ and a binding energy of $162.0 \mathrm{eV}$, typical for the $\mathrm{S} 2 p_{3 / 2}$ level of the thiolate species [34]. Likewise the $2 p_{3 / 2}$ linewidth of $0.66 \mathrm{eV}$ is independent of SAM composition and compares well with a high-resolution XPS study of Az6 [32]. This indicates that, independent of the mixture in solution, surface contaminations are displaced during the self-assembly process and the goldsulphur bond is little affected by lateral inhomogeneities.

$\mathrm{XP}$ spectra of the $\mathrm{C} 1 s$ region are depicted in Fig. 2a. The concentration of TF-Az6 in solution decreases from top to bottom, while the concentration of CN-Az6 increases accordingly. The first spectrum shows the C1s XPS of a pure TF-Az6 SAM, the last belongs to a pure CN-Az6 SAM. Comparison of the two spectra reveals several chemically shifted emission lines. For TF-Az6 we find the maximum of the main line at $284.65 \mathrm{eV}$, which contains contributions of the aliphatic alkane chain and the aromatic azobenzene chromophore. The peaks at $286.3 \mathrm{eV}$ and $292.2 \mathrm{eV}$ are attributed to the chemically shifted carbon atoms of the $\mathrm{C}-\mathrm{O}-\mathrm{C}$ and $\mathrm{CF}_{3}$ groups, respectively [32]. The similar peak intensities of these components are compatible with the upright orientation of the molecule, and are attributed to the larger damping of the $\mathrm{C} 1 s$ signal of the C-O$\mathrm{C}$ linker by the overlying chromophore compared to the end group at the film surface (cf. Fig. 1 and Section 3.2). This observation also implies that the C1s main peak is dominated by the carbon XPS signal of the chromophore with a minor contribution from the alkane chain.

With increasing $\mathrm{CN}-\mathrm{Az} 6$ concentration the maximum of the main line shifts to $285.0 \mathrm{eV}$. The low-energy onset remains at constant binding energy and the main peak broadens.

A similar shift is observed for the C-O-C peak. Since its linewidth is broadened 

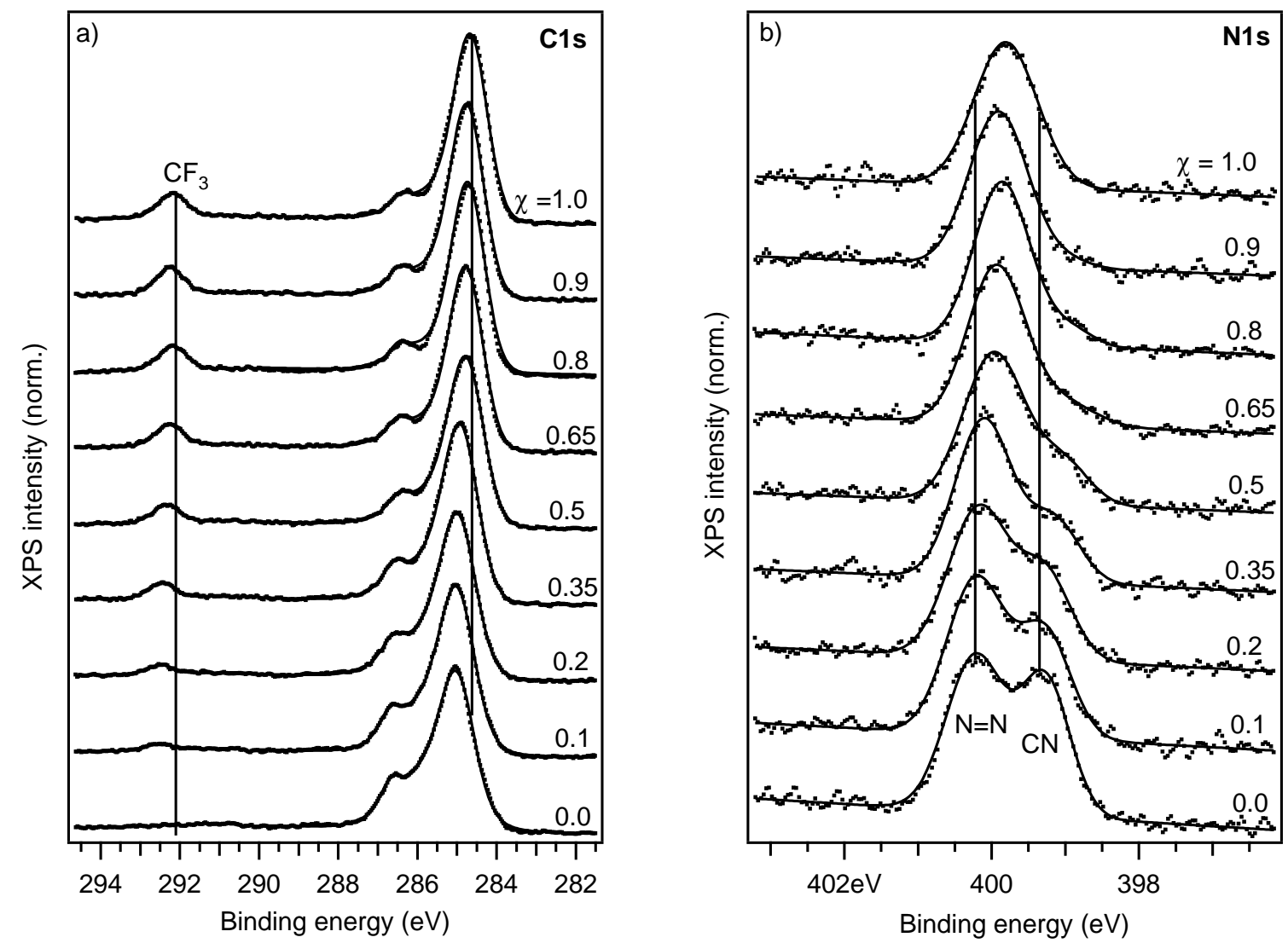

Figure 2. XP spectra of SAMs on Au/mica with varying TF-Az6 molar fraction $\chi$ and CN-Az6 molar fraction $(1-\chi)$. Solid lines are fits to the data as described in Subsection 2.2. Vertical lines reveal peak shifts, detailed in Fig. 4.

a) The $\mathrm{C} 1 s \mathrm{XP}$ spectra contain contributions from the $\mathrm{C}-\mathrm{H}$ alkane chain and $\mathrm{C}=\mathrm{C}$ chromophore (main peak) and the chemically shifted $\mathrm{C}-\mathrm{O}, \mathrm{C} \equiv \mathrm{N}$ and $\mathrm{CF}_{3}$ components. b) In the $\mathrm{N} 1 s \mathrm{XP}$ spectra the peaks stem from the $\mathrm{N}=\mathrm{N}$ unit present in both molecules and the $\mathrm{CN}$ group of $\mathrm{CN}-\mathrm{Az} 6$.

and its intensity is approximately doubled, the XPS peak must in addition contain the $\mathrm{C} 1 s$ contribution from the cyano end group. Obviously no $\mathrm{CF}_{3}$ component is observed for the pure CN-Az6 SAM. The weak background between 288 and $293 \mathrm{eV}$ is attributed to shake-up satellites of the azobenzene chromophore.

From the series of $\mathrm{C} 1 s$ XP spectra we can already deduce some details of the SAM composition. The increase of the XPS signal of the $\mathrm{CF}_{3}$ component is similar to the increase in TF-Az6 concentration in solution. Therefore the mixture in solution is roughly reproduced at the surface. Furthermore, all core levels shift to lower binding energy with increasing $\mathrm{CF}_{3}$ concentration.

Because the chromophores are electronically decoupled from the substrate the core levels are not pinned to the gold fermi level, but depend on the local potential [35]. This is influenced by the molecular environment of the respective species. Since we observe a similar shift for all components of all chromophores, the contribution of the molecular environment to the local potential must be similar for both the TF-Az6 and the CN-Az6 


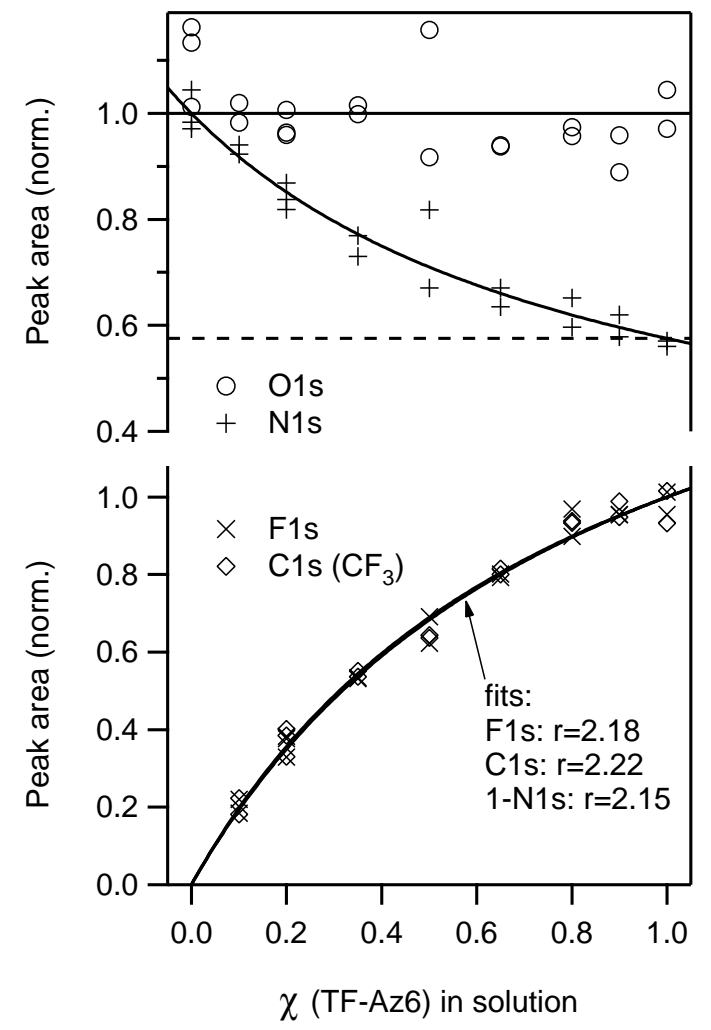

Figure 3. XPS intensities of the $\mathrm{O} 1 s, \mathrm{~N} 1 s, \mathrm{~F} 1 s$ and $\mathrm{C} 1 s-\mathrm{CF}_{3}$ core levels as a function of TF-Az6 fraction $\chi$ in solution. All spectra were normalized to the intensity of the $4 f$ core levels of the gold substrate and the maximum value is set to 1 . Accordingly the normalized peak area in the lower panel corresponds to the TF-Az6 fraction on the surface. Solid lines are fits to Eq. 3. For the N1s intensity the constant background of the azo group has been subtracted.

molecules. This can only happen if cyano- and trifluoromethyl-terminated molecules are in close proximity, of order $3 \mathrm{~nm}$. On the contrary, phase separation into islands of TFAz6 and CN-Az6 would lead to a constant, concentration-invariant binding energy of, e.g., the $\mathrm{CF}_{3}$ XPS line (see Fig. 6).

The latter conclusion is corroborated by the series of $\mathrm{N} 1 s$ XP spectra depicted in Fig. 2b. The first spectrum corresponds to the TF-Az6 SAM and we observe only the N1s component of the azo group at $399.8 \mathrm{eV}$. The last spectrum of the series shows a double peak structure at $400.2 \mathrm{eV}$ and $399.3 \mathrm{eV}$, which we assign to the chemically shifted N1s core levels of the azo and cyano groups of CN-Az6. As is evident from Fig. 2b, both peaks shift with increasing TF-Az6 concentration to higher binding energies. This confirms that the dipole moment of the trifluoromethyl end group also alters the local potential of the cyano end group and thus implies adsorption of TF-Az6 and CN-Az6 molecules on adjacent lattice sites.

Finally we quantify the development of the surface concentrations and the corresponding peak shifts for various core levels. Figure 3 summarizes the XPS 


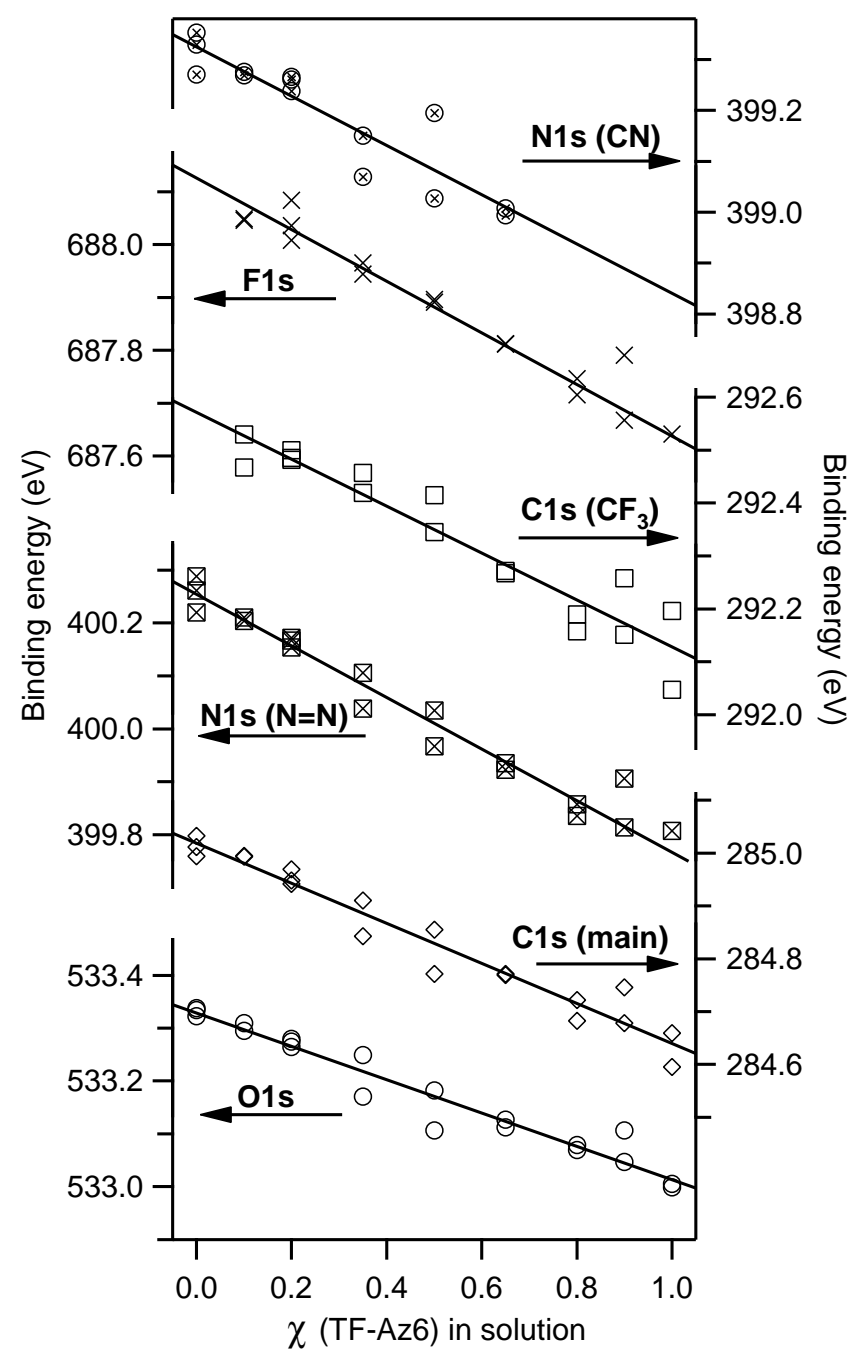

Figure 4. XPS binding energies of the $\mathrm{F} 1 s, \mathrm{C} 1 s, \mathrm{~N} 1 s$ and $\mathrm{O} 1 s$ core levels as a function of TF-Az6 fraction $\chi$ in solution. The $\mathrm{C} 1 s \mathrm{XP}$ spectra include the maximum position of the main peak and of the chemically shifted $\mathrm{CF}_{3}$ component. Solid lines are a fit assuming a linear dependence of the binding energies on TF-Az6 fraction.

intensities of the $\mathrm{O} 1 s, \mathrm{~F} 1 s, \mathrm{C} 1 s-\left(\mathrm{CF}_{3}\right)$, and $\mathrm{N} 1 s$ core levels as a function of $\mathrm{TF}$ Az6 fraction $\chi$ in solution. All intensities have been normalized to 1 for the single component SAMs. Since each molecule contains one oxygen atom, the uniformity of the O1s XPS intensity, which has a standard deviation below \pm 0.08 , proves a constant coverage independent of the SAM composition. As a consequence the normalized areas of the $\mathrm{F} 1 s$ and $\mathrm{C} 1 s\left(\mathrm{CF}_{3}\right)$ peak directly represent the surface fraction of the TF-Az6 species. For all mixtures we find a higher fraction of TF-Az6 molecules on the surface than in solution. The N1s integral consists of the contribution of the cyano group on a constant background from the azo group. Thus the variation above this background reflects the $\mathrm{CN}-\mathrm{Az} 6$ fraction on the surface. It decreases accordingly towards higher 
TF-Az6 fractions.

The preferential adsorption can be nicely modelled assuming that after an immersion time of 24 hours thermodynamic equilibrium is reached. Since the total coverage is independent of the SAM composition, the exchange reaction of the two adsorbed compounds $\mathrm{A}$ and $\mathrm{B}$ with the solution can be written as

$$
A^{s o l}+B^{a d s} \rightleftharpoons A^{a d s}+B^{s o l}
$$

with the equilibrium constant

$$
K_{e q}=\frac{c_{A}^{s o l} c_{B}^{a d s}}{c_{A}^{a d s} c_{B}^{s o l}}
$$

Expanding with the total concentrations in solution $\left(c_{A}^{s o l}+c_{B}^{s o l}\right)$ and on the surface $\left(c_{A}^{a d s}+c_{B}^{a d s}\right)$, we replace the concentrations $c$ by molar fractions $\chi$.

Using $\chi_{B}^{\text {sol } / a d s}=1-\chi_{A}^{\text {sol } / a d s}$ we get for the XPS intensity $I_{A}$ of the adsorbed species A:

$$
I_{A} \propto \chi_{A}^{a d s}=\frac{K_{e q} \chi_{A}^{s o l}}{1+\left(K_{e q}-1\right) \chi_{A}^{s o l}}
$$

The independent fits of Eq. 3 to the peak integrals of $\mathrm{F} 1 s$ and $\mathrm{C} 1 s\left(\mathrm{CF}_{3}\right)$ as well as $[1-\mathrm{N} 1 s(\mathrm{CN})]$ (solid lines in Figure 3 ) all yield an average equilibrium constant of $K_{e q}=2.2 \pm 0.2$.

Thus for a 1:1 mixture of TF-Az6 and CN-Az6 molecules in solution, the ratio of adsorbed molecules is about $2: 1$. The interplay of attractive van der Waals interactions and dipole-dipole repulsions may lead to this preferential adsorption of TF-Az6. Altogether, the XP spectra demonstrate that we can quite accurately adjust any surface concentration via an appropriate mixture of the molecules in solution and immersion for 24 hours at room temperature.

Figure 4 gives a survey of the XPS peak positions. All XPS lines of the chromophore shift to lower binding energy with increasing TF-Az6 fraction suggesting a true mixture of the two molecules on the surface. Moreover, the linear fits result in comparable slopes for core-levels located at the end group or the azobenzene moiety of either molecule. For the F1s and $\mathrm{C} 1 s$ core holes of the trifluoromethyl end group we find slopes of $-0.49 \pm 0.04 \mathrm{eV}$ and $-0.44 \pm 0.04 \mathrm{eV}$, for the $\mathrm{N} 1 s$ core levels of the cyano and the azo group $-0.48 \pm 0.06 \mathrm{eV}$ and $-0.49 \pm 0.02 \mathrm{eV}$. The $\mathrm{C} 1 s$ main peak, which is dominated by the phenyl rings but also contains contributions of the alkyl chains, shifts accordingly by $-0.38 \pm 0.02 \mathrm{eV}$. Finally, the $1 \mathrm{~s}$ core hole of the oxygen bridge between the azobenzene chromophore and the alkane chain shows the smallest change in binding energy $(-0.32 \pm 0.02 \mathrm{eV})$ as a function of the TF-Az6 concentration. As already mentioned, the low-energy onset of the $\mathrm{C} 1 s$ XP spectrum and the $\mathrm{S} 2 p$ core level stay at constant binding energy.

Obviously the shift of the XPS binding energy with TF-Az6 concentration is within error bars constant across the extended electronic $\pi$-system of the chromophore. We therefore conclude that the mixture of TF-Az6 and CN-Az6 dipole moments, which point towards the surface (see Section 3.2), modifies the local potential in front of the 


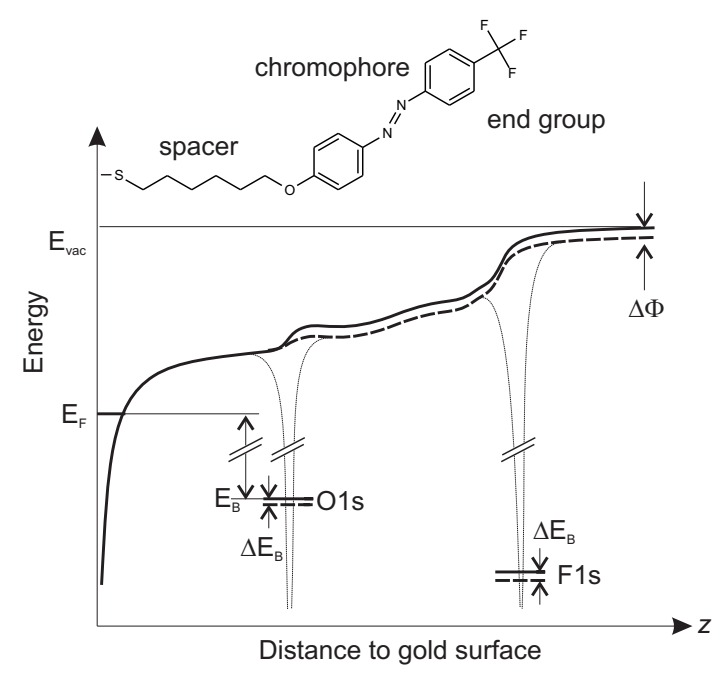

Figure 5. Sketch of the TF-Az6 molecule and the local potential in front of the surface. Note that the XPS binding energies $E_{B}$ are referenced to the Fermi level $E_{F}$ of the gold sample. The position of the vacuum level $E_{v a c}$ depends on the concentration of TF-Az6 and CN-Az6 molecules. A change of the concentration alters the sample workfunction by $\Delta \Phi$. Locally this corresponds to a drop of the potential at the TF and $\mathrm{CN}$ end groups of the aromatic chromophore and at the C-O-C linker to the aliphatic alkane chain. The change in local potential leads to the concentration-dependent shift of the XPS binding energies.

surface. Calculations yield a larger dipole moment of 6.76 Debye for TF-Az6 [36] as compared to 4.93 Debye for CN-Az6 [22]. Since the alkane chain effectively decouples the chromophore from the surface, the core levels of the chromophore become hooked on the local potential [35]. The potential drop will mainly occur across the alkane chain, which exhibits the highest resistance for carrier transport. This likewise explains the smaller shift of the $\mathrm{O} 1 s$ core level of the $\mathrm{C}-\mathrm{O}-\mathrm{C}$ linker and the $\mathrm{C} 1 s$ main peak, which contains the contribution of the aliphatic spacer. The increase of the TF-Az6 fraction and concomitant decrease of CN-Az6 coverage with their different dipole moments lead to an increase of the local potential at the film-vacuum interface and a corresponding shift of all electronic levels [37, 38]. A simple model of a one-dimensional surface potential is sketched in Fig. 5. Since the azobenzene moiety including the oxygen atom is conjugated, the change of the potential should extend over the whole chromophore. When referenced to the Au Fermi level, the increase of the local potential leads to a decrease of the core level binding energies. Here we interpret the shift of the core levels as a change of the ground-state potential. We note that in addition the XPS binding energies may be modified by concentration-dependent polarization screening of the XPS final state. Nevertheless, the distinct shifts of the core levels of all the chromophores clearly demonstrate a short-range mixture of CN-Az6 and TF-Az6 molecules.

To further investigate the lateral texture of the mixed SAMs we solve a dipole model on a square grid of $100 \times 100$ adsorption sites with periodic boundary conditions [20, 39]. We assume equal adsorption probabilities of species $A$ and $B$, but allow for lateral 


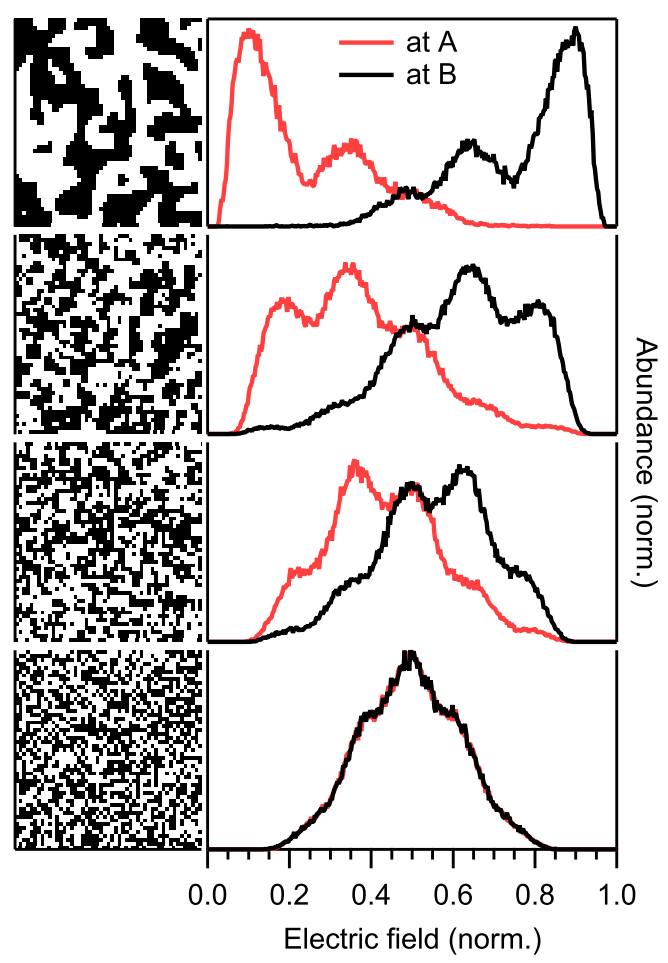

Figure 6. Left panel: $100 \times 100$ adsorption sites with different, representative arrangements of adsorbed species $A$ and $B$ illustrated by black and white squares. The average island size of equivalent species decreases from top to bottom (see text). Right panel: Distribution of the electric field strength calculated in a dipole model for the molecular arrangements depicted in the left panel. Data of the electric field strength are averaged over 30 runs.

interactions among the adsorbates. The intermolecular interactions lead, depending on their hierarchy, to either island formation $A A \sim B B>A B$ or statistical mixtures $A A \sim B B \sim A B$ of species $A$ and $B$. Varying these constraints for a $1: 1$ mixture of molecules we obtain the distinct patterns of molecules $A$ and $B$ represented in the right panel of Fig. 6 by black and white squares, respectively. The four cases resemble textures from phase separation (top) via small island formation to a statistical mixture (bottom). The mixtures are obtained by simulating the formation of the SAM by a statistical occupation of the grid positions including exchange reactions within a Monte Carlo simulation. For each of these configurations we calculate (averaged over 30 runs) the electric field at the position of each molecule in a dipole model. Thereby the dipole field is calculated at the position of an individual molecule caused by all its neighbours. The resulting field distributions are shown in the left panel of Fig. 6. The additional electric field will modify the local potential and is thus a measure of the XPS peak-shift. The relative field strengths for homogenous layers of species $A$ and $B$ are set to 0 and 1, respectively. This difference reflects the significant shift of the XPS lines by $0.4 \mathrm{eV}$ between the pure TF-Az6 and CN-Az6 SAMs (see Fig. 4). For large islands (top panel in Fig. 6) we observe clearly separated maxima close to the positions of pure species $A$ and 
$B$. With decreasing island size, the number of atoms at the boundary between islands increases. As atoms at the boundary experience an averaged dipole field between 0 and 1, the field distribution smears over. For even smaller island sizes almost almost every molecule has neighbors of the other species and the field distribution finally centers at 0.5 for the case of random mixing (bottom panel in Fig. 6).

In the XPS data we see a continuous shift of all peak positions when varying the concentration of TF-Az6 vs. CN-Az6 and negligible linewidth broadening with respect to the pure layers. From the above simulation it becomes obvious that this observation is not compatible with island growth even if the average island's diameter is as small as 10 next neighbour distances $(\sim 30 \AA)$. This sensitivity of XPS to the local composition is due to the short range of the dipole interaction. While it decays with distance $d$ as $d^{-3}$, the number of molecules in subsequent coordination shells increases proportional to $d$. Therefore, even without screening (depolarization), all second next neighbours account for only one quarter of the nearest neighbours' field. We conclude that the XPS data strongly support random mixing of species $A$ and $B$ as depicted in the bottom left panel of Fig. 6.

\subsection{Molecular orientation}

As the substituents of the TF-Az6 and CN-Az6 molecules have different dipole moments, the molecular orientation of the azobenzene moieties may differ between the pure and the mixed SAMs. The orientation of the chromophores can be extracted from the polarization dependence of the $\mathrm{C} 1 s$ and N1s NEXAFS. For the $1 s$ to $\pi^{*}$ transitions of the chromophore the orientation of the transition dipole moment (TDM) with respect to the surface normal $\vec{n}$ can be evaluated from the NEXAFS contrast. For these vectorlike orbitals the absorption is proportional to $\cos ^{2} \varphi$ where $\varphi$ denotes the angle between the electric field vector $\overrightarrow{\mathcal{E}}$ and the TDM. If the different azimuthal orientations of the molecule appear with equal probability in at least a three-fold symmetry, e.g., the three rotational domains of the $\mathrm{Au}(111)$ terraces on mica, the angle-dependent part of the $\mathrm{X}$-ray absorption is given by

$$
I_{\mathrm{v}} \propto \sin ^{2} \alpha\left(1-3 \cos ^{2} \varphi \cos ^{2} \beta\right)+2 \cos ^{2} \varphi \cos ^{2} \beta .
$$

Here $\beta=20^{\circ}$ is the fixed grazing incidence angle of the X-ray beam and $\varphi$ the angle of the field vector $\overrightarrow{\mathcal{E}}$ relative to the plane of incidence, defined by the X-ray beam and the surface normal $\vec{n}$. The angle $\alpha$ represents the orientation of the TDM relative to $\vec{n}$ (see Fig. 1). By measuring NEXAFS spectra for $\varphi=18^{\circ}$ and $\varphi=90^{\circ}, \alpha$ can be determined

$$
\frac{1}{\sin ^{2} \alpha}=\frac{3}{2}+\frac{1}{2 \cos ^{2} \beta} \cdot \frac{I_{\mathrm{v} 1}-I_{\mathrm{v} 2}}{I_{\mathrm{v} 2} \cos ^{2} \varphi_{1}-I_{\mathrm{v} 1} \cos ^{2} \varphi_{2}} .
$$

As the polarization $P$ of the synchrotron radiation is only perfectly linear in the plane of the storage ring, we have to take into account a certain proportion $1-P$ of light with orthogonal polarization. Therefore the measured absorption corresponds to

$$
I_{\mathrm{v}}(\varphi, P)=P \cdot I_{\mathrm{v}}(\varphi)+(1-P) \cdot I_{\mathrm{v}}\left(\varphi-90^{\circ}\right) .
$$


In this case the angular orientation $\alpha$ of the molecular TDM is numerically evaluated.

NEXAFS spectra recorded at the carbon and nitrogen edges are shown in Figs. 7 and 8, respectively, for SAMs of TF-Az6 and CN-Az6, as well as for a 1: 1 mixture of the molecules on gold on mica. The spectra are normalized to the photon flux and are aligned at the pre-edge below the first transition and at $330 \mathrm{eV}$ and $430 \mathrm{eV}$, respectively. For these photon energies well above the ionization threshold, no significant resonances are observed and the measured signal can be assumed to be independent of the molecular orientation. NEXAFS spectra were recorded in Auger yield for $s$ and $p$-polarization $\left(\varphi=90^{\circ}\right.$ and $\left.18^{\circ}\right)$ and for the magic angle $\left(\varphi \sim 52^{\circ}\right.$, not shown). Overall, the pronounced polarization dependence signifies a high degree of molecular orientation within the SAMs. The contrast is nearly independent of the end group, and the absorption spectra of the mixed SAM can be reproduced in detail by simply adding up $50 \%$ of the appropriate TF-Az6 and CN-Az6 spectra. This indicates that the average orientation of the molecules is little affected by the end group. The NEXAFS is dominated by transitions into the $\pi^{*}$-resonances of the azobenzene moiety. The corresponding lowest unoccupied molecular orbitals $(\mathrm{LUMO}+\mathrm{n})$ are depicted in Fig. 9 for the TF-Az6 molecule.

At the carbon edge the dominant structure extends from 284.0 to $286.0 \mathrm{eV}$. It is assigned to transitions from the $\mathrm{C} 1 s$ to the $\pi^{*}$ LUMO and to the higher lying, nearly degenerate $\mathrm{LUMO}+1$ and $\mathrm{LUMO}+2$. For tetra-tert-butyl-azobenzene (TBA) adsorbed on $\mathrm{Au}(111)$ the $\pi^{*} \mathrm{LUMO}$ resonance at $284.0 \mathrm{eV}$ is clearly separated from transitions into the $\mathrm{LUMO}+1,2$ at $285.3 \mathrm{eV}$ [40]. As these orbitals extend over the two phenyl rings and the cyano group, the broadening of the $\pi^{*} \mathrm{LUMO}$ and LUMO+1,2 structures for CNAz6 and TF-Az6 is attributed to the different binding energies of the carbon atoms (cf. Fig. 2a). The larger splitting of the corresponding C-O and CN XPS peaks must stem from distinct screening of the core-ionized XPS final-states compared to the core exciton probed in NEXAFS. In contrast to the XP spectra, the $\mathrm{C} 1 s$ to $\pi^{*}$ transition energy does not significantly alter with SAM composition, since intermolecular excitations are not affected by the change of the local work function (cf. Fig. 5). From the strong polarization contrast of the $\mathrm{C} 1 s$ to $\pi^{*}$ transition, the mean tilt angle $\alpha_{1, C}$ of the TDM with respect to the surface normal can be determined (cf. Fig. 1). The average tilt angle of the phenyl rings $\alpha_{1, C}=71-75^{\circ}$ are listed in Tab. 1 . The tilt angles of the chromophore are comparable for the three different SAMs within the error of $\pm 5^{\circ}$.

For CN-Az6 a second absorption structure occurs at $286.5 \mathrm{eV}$ and exhibits a vibrational progression. Following studies of biphenylnitrile-based SAMs [41, 42, 43] we attribute this transition to the $\pi^{*}$-orbital at the cyano group. The transition energy and vibrational fine structure are comparable to the $\pi^{*}$ resonance observed in SAMs of nitrilesubstituted alkanethiolate (CN-C16) [44]. The energetic spacing is in agreement with the value of $250 \mathrm{meV}$ calculated for the $\mathrm{C}^{*} \mathrm{~N}$ stretch vibration [45, 46]. The $\pi^{*}$-orbital and corresponding TDM are localized at the cyano group and oriented perpendicular to the molecular plane of the azobenzene chromophore. The weak polarization dependence indicates that the tilt angle $\alpha_{2}$ is close to the magic angle. 


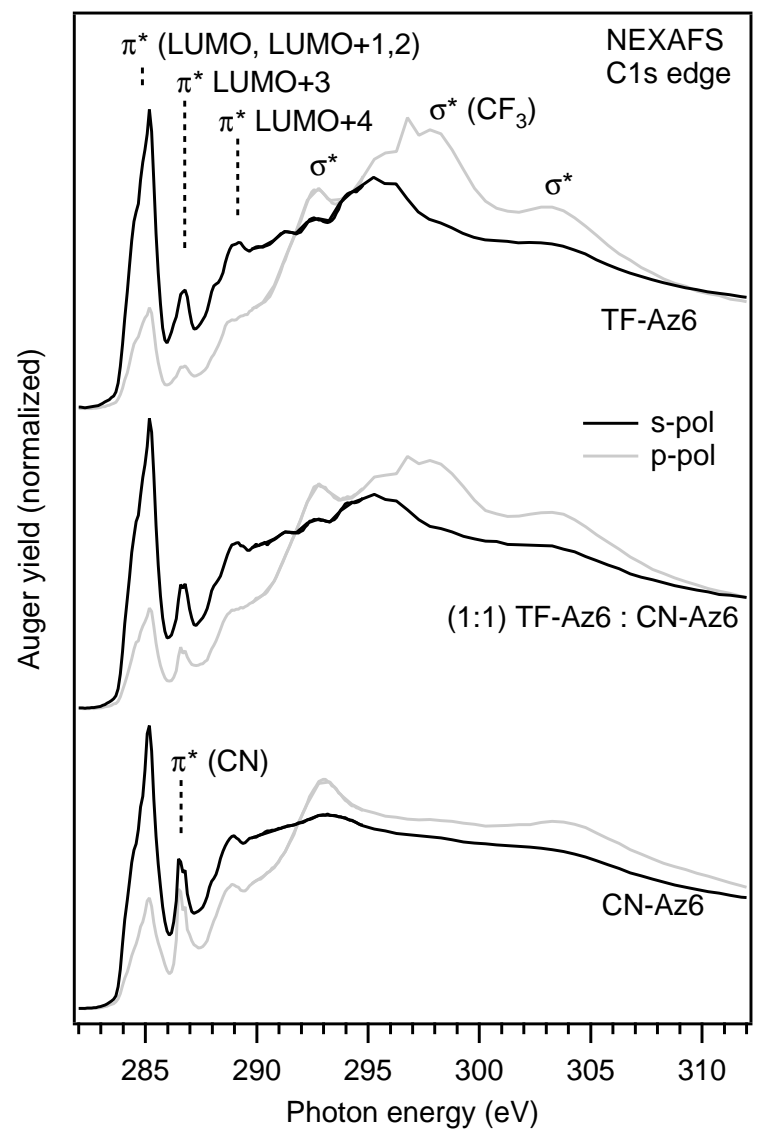

Figure 7. C1s NEXAFS spectra of TF-Az6, CN-Az6, and a $1: 1$ mixture. The sample was rotated around the X-ray beam axis at a fixed angle of incidence of $20^{\circ}$ with respect to the surface plane. For $s$ - and $p$-polarization the field vector was perpendicular $\left(\varphi=90^{\circ}\right)$ and nearly parallel $\left(\varphi=18^{\circ}\right)$ to the plane of incidence, respectively.

The remaining absorption peaks in the energy range up to $5 \mathrm{eV}$ above the LUMO and LUMO+1,2 resonances are assigned to excitations to higher lying $\pi^{*}$ orbitals of the aromatic system and to mixed C-H valence / Rydberg states. The transition at $286.7 \mathrm{eV}$ observed for TF-Az6 is assigned to the LUMO+3 resonance, since the polarization contrast resembles that of the main $\pi^{*}$ resonance and the TDM is thus oriented perpendicular to the ring plane. In the $\mathrm{C} 1 s$ NEXAFS of $\mathrm{CN}-\mathrm{Az} 6$ we cannot isolate this transition as an individual peak, but it must contribute to the overall background, which is larger for $s$-polarization in the respective photon-energy range. The shoulder at around $288.0 \mathrm{eV}$ is observed in all $\mathrm{C} 1 s$ NEXAFS spectra and is characteristic for alkane SAMs. It is assigned to transitions into the $\mathrm{C} 1 s \sigma^{*}-(\mathrm{C}-\mathrm{H})$ and $\mathrm{C} 1 s \pi^{*}-(\mathrm{C}-\mathrm{H})$ resonances of the alkane chain $[47,48]$. The $\sigma^{*}$ and $\pi^{*} \mathrm{TDMs}$ of the $\mathrm{CH}_{2}$ groups are oriented within the $\mathrm{H}-\mathrm{C}-\mathrm{H}$ plane and the polarization dependence is compatible with an upright orientation of the alkane chain. The resonance at $289.0 \mathrm{eV}$ is attributed to the $\mathrm{C} 1 \mathrm{~s}$ to $\mathrm{LUMO}+4$ transition. Its polarization dependence resembles that of the main $\pi^{*}$ resonance. The $\mathrm{LUMO}+4$ is mainly located at the azo group and the inner phenyl ring (see Fig. 9), and 


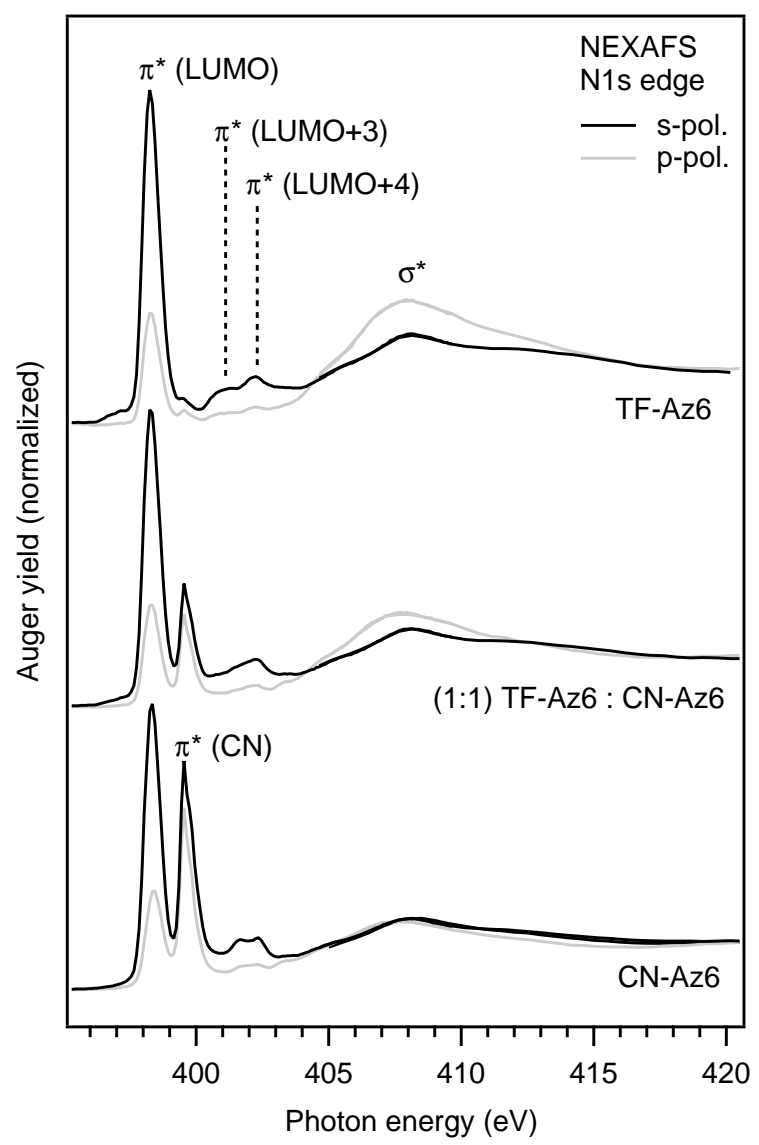

Figure 8. N1s NEXAFS spectra of TF-Az6, CN-Az6, and a 1 : 1 mixture. Measurement geometry as in Fig. 7.
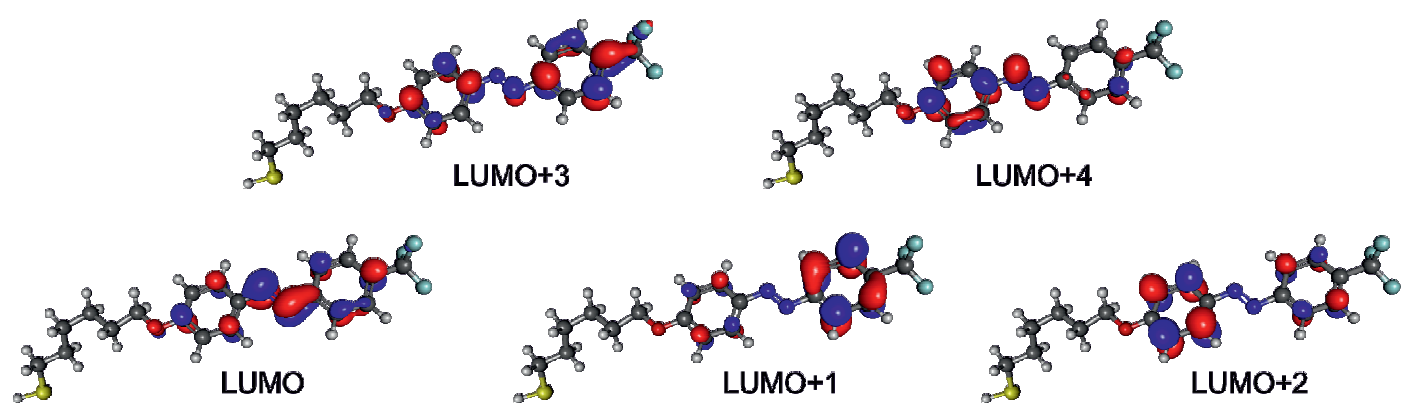

Figure 9. Isodensity contours of the lowest unoccupied molecular orbitals $(\mathrm{LUMO}+n)$ of TF-Az6 (from calculations at the DFT-B3LYP level, see ref.[32] for further details). 
Table 1. Tilt and twist angles of the transition dipole moments sketched in Fig. 1 for TF-Az6, CN-Az6 and a $1: 1$ mixture of the molecules. Within the error bar of $\pm 5^{\circ}$ the molecular orientation is identical in the pure and mixed SAMs. Values in parentheses are calculated according to $\cos \alpha_{2}=\sin \vartheta \cdot \sin \gamma$ and $\cos \alpha_{1}=\sin \vartheta \cdot \cos \gamma$.

\begin{tabular}{lccccc}
\hline Molecule & $\alpha_{1, C}$ & $\alpha_{1, N}$ & $\alpha_{2, N}$ & $\gamma$ & $\vartheta$ \\
\hline TF-Az6 & $74^{\circ}$ & $74.5^{\circ}$ & $\left(65^{\circ}\right)$ & $\left(57^{\circ}\right)$ & $30^{\circ}$ \\
CN-Az6 & $71^{\circ}$ & $74^{\circ}$ & $59^{\circ}$ & $\left(58^{\circ}\right)$ & $\left(36^{\circ}\right)$ \\
mixed SAM & $75^{\circ}$ & $72.5^{\circ}$ & $60^{\circ}$ & $\left(60^{\circ}, 50^{\circ}\right)$ & $27^{\circ},\left(33.5^{\circ}\right)$ \\
\hline
\end{tabular}

its position is thus little affected by the different end groups.

The higher-lying, broad resonances at 293.0 and $303.0 \mathrm{eV}$ are attributed to shape resonances. As expected these $\sigma^{*}$ resonances of the chromophore exhibit similar polarization contrast for all SAMs and opposite to the $\pi^{*}$ resonances.

The C1s NEXAFS spectra of TF-Az6 show in addition pronounced resonances in the photon energy range of 294.0 to $300.0 \mathrm{eV}$. These absorption features are missing in the NEXAFS of CN-Az6 in Fig. 7. They are attributed to excitations involving the C1s level of the $\mathrm{CF}_{3}$ group of TF-Az6, which is chemically shifted by about $8.0 \mathrm{eV}$ with respect to the $\mathrm{C} 1 s$ core levels of the aromatic rings (cf. Fig. 2a). As discussed in detail in Ref. [20], the main resonances can be assigned in analogy to the NEXAFS of hexafluoroethane $[49,50]$. The polarization dependence of the $\mathrm{C} 1 s$ to $\sigma^{*}(\mathrm{C}-\mathrm{C})$ resonance at $298.3 \mathrm{eV}$ allows us to determine the tilt angle $\vartheta$ of the $\mathrm{C}_{-} \mathrm{CF}_{3}$ end group (cf. Fig. 1). It is evaluated using the 'building-block' scheme, i.e., the CN-Az6 NEXAFS spectrum contains all but the $\mathrm{CF}_{3}$ related transitions. Subtracting the CN-Az6 from the TF-Az6 NEXAFS spectrum to remove the background from the broad carbon shape resonances we obtain a tilt angle $\vartheta$ of the molecular axis of around $30^{\circ}$ (see Tab. 1).

The average tilt angles of the azobenzene chromophore can be independently determined at the nitrogen edge. Corresponding NEXAFS spectra of TF-Az6, CNAz6 and the 1: 1 mixture are depicted in Fig. 8. The N1s to LUMO $\pi^{*}$ transition is observed at photon energies of 398.3 and $398.4 \mathrm{eV}$ for TF-Az6 and CN-Az6 and shifts to the mean value for the mixture. This small energetic difference points to a small shift of the $\pi^{*}$ resonance position between the azo and cyano groups. Note that the XP spectra of the core-ionized N1s atoms show a bigger shift of $0.9 \mathrm{eV}$ between these groups (cf. Fig. 2b). In contrast to the carbon edge the N1s to LUMO+1 and $\mathrm{LUMO}+2$ transitions have negligible dipole moment, since the valence orbitals exhibit little probability density at the azo group (see Fig. 9). From the polarization dependence of the N1s to LUMO transition we obtain the tilt angles $\alpha_{1, N}$ of the TDM of the azo group. As listed in Tab. 1 these are comparable to the tilt angles of the phenyl rings $\alpha_{1, C}$ indicating that the azobenzene chromophores adsorb in the trans configuration. The transitions at 401.0 and $401.6 \mathrm{eV}$ in TF-Az6 and CN-Az6 are attributed to the 
LUMO +3 resonance, respectively. As depicted in Fig. 9 the LUMO+3 extends over the carbon atom of the trifluoromethyl end group, which can explain the shift to lower energies of the corresponding resonance. The transition at $402.3 \mathrm{eV}$ is attributed to the $\mathrm{LUMO}+4$ resonance. As already mentioned this orbital is mainly located at the azo group and the inner phenyl ring. Therefore the resonance position is not affected by the substituent.

For CN-Az6 the additional resonance at $399.6 \mathrm{eV}$ labelled $\pi^{*}(\mathrm{CN})$ in Fig. 7 is attributed to the transition into the $\pi^{*}$ orbital localized at the cyano group [43]. Resonance energy and vibrational fine structure are again very similar to CN-C16 SAMs [44]. Even though only one nitrogen atom per molecule contributes to this resonance, its intensity is only slightly weaker than that of the $\pi^{*}$ LUMO where three nitrogen atoms contribute. This reflects the strong localization of the orbital at the cyano group with the nodal plane perpendicular to the LUMO, which is in contrast delocalized over the whole chromophore. From the polarization dependence of the $\pi^{*}(\mathrm{CN})$ resonance we obtain the tilt angle $\alpha_{2, N}$ of $59^{\circ}$.

The twist and tilt angles of the chromophore and end group are related via

$$
\begin{aligned}
& \cos \gamma=\cos \alpha_{1} / \sin \vartheta, \\
& \tan \gamma=\cos \alpha_{2} / \cos \alpha_{1}, \\
& \sin ^{2} \vartheta=\cos ^{2} \alpha_{1}+\cos ^{2} \alpha_{2} .
\end{aligned}
$$

The comparison in Tab. 1 demonstrates that both the measured and calculated twist and tilt angles of all the molecules are comparable and, within the error, independent of SAM composition. When comparing the nitrogen $\sigma^{*}$ resonance at $403 \mathrm{eV}$ we notice a different polarization dependence between TF-Az6 and CN-Az6. While the former shows the expected strong polarization contrast opposite to the $\pi^{*}$ LUMO resonance, the latter lacks this contrast. We conclude, that for $\mathrm{CN}-\mathrm{Az} 6$ the polarization dependence of the two $\sigma^{*}$ orbitals of the azo $(\mathrm{N}=\mathrm{N})$ and cyano $(\mathrm{CN})$ groups cancel. Given the orientation of the chromophore, this is only possible if both TDMs take up clearly different tilt angles. There are two possible orientations of the chromophore which result from a $\gamma=180^{\circ}$ twist around the C-O-C linker. While this leaves all the angles in Tab. 1 unchanged, the azo bridge is either oriented parallel to the cyano group or parallel to the surface plane (cf. Fig. 1). At least for CN-Az6 the latter case must be realized, and it is most likely that this is the preferential adsorption geometry in all SAMs.

\subsection{Summary}

The composition and geometrical structure of SAMs of alkanethiolates carrying azobenzene photoswitches were studied by XPS and NEXAFS spectroscopy. The orientation of the molecules is independent of the trifluoromethyl or cyano end group attached in the para position (TF-Az6 and CN-Az6). The main axis of the azobenzene chromophore is tilted with respect to the surface normal by $30^{\circ}$ while the molecular plane of the chromophore is twisted by $60^{\circ}$. This orientation is determined by measuring both 
the tilt and twist angle of the $\mathrm{CF}_{3}$ and $\mathrm{CN}$ end groups in the pure SAMs and a $1: 1$ mixed SAM. For all SAMs we find the same binding energy and width of the sulphur $2 p$ XPS lines. This indicates that the aliphatic linker, a hexane-thiol, attached via an oxygen bridge in para position to the azobenezene, is flexible enough to adjust to the rigid gold-sulphur bond. Interestingly, the NEXAFS measurements suggest that the azo bridge of the chromophore, which adsorbs in the trans configuration, is oriented parallel to the surface. This orientation implies substantial steric hindrance when switching the molecule into the cis conformer.

The fact that the end groups barely affect the molecular orientation certainly contributes to the satisfactory miscibility of TF-Az6 and CN-Az6 molecules at the gold surface. Changing the concentration of the molecules with trifluoromethyl vs. cyano end groups in solution allows us to adjust the concentration of each species in the SAM between 10 and $90 \%$. The continuous shift of the XPS lines of the chromophore with concentration thereby suggests that the SAM consists of a homogenous mixture of TFAz6 and CN-Az6 molecules.

Combining different end groups allows modification of the optical absorption band of the chromophore. By this means excitonic coupling among neighbouring chromophores may be suppressed. Moreover, by overlapping emission and absorption bands of the two modified chromophores, light harvesting and funneling in a SAM may become feasible.

\section{Acknowledgments}

We thank Joachim Leistner for UPLC analysis of the azo compounds. This work has been supported by the Deutsche Forschungsgemeinschaft through Sonderforschungsbereich 658 .

\section{References}

[1] Ulman A, Chem. Rev. 96, 1533 (1996).

[2] DiBenedetto S. A, Facchetti A, Ratner M. A, and Marks T. J, Adv. Mater. 21, 1407 (2009).

[3] Samanta D and Sarkar A, Chem. Soc. Rev. 40, 2567 (2011).

[4] Love J. C, Estroff L. A, Kriebel J. K, Nuzzo R. G, and Whitesides G. M, Chem. Rev. 105, 1103 (2005).

[5] Schreiber F, Prog. Surf. Sci. 65, 151 (2000).

[6] Schwartz D. K, Annu. Rev. Phys. Chem. 52, 107 (2001).

[7] Zharnikov M and Grunze M, J. Phys.:Condens.Matter 13, 11333 (2001).

[8] Shaporenko A, Brunnbauer M, Terfort A, Grunze M, and Zharnikov M, J. Phys. Chem. B 108, 14462 (2004).

[9] Cyganik P, Buck M, Azzam W, and Wöll C, J. Phys. Chem. B 108, 4989 (2004).

[10] Cyganik P, Buck M, Strunskus T, Shaporenko A, Wilton-Ely J. D. E. T, Zharnikov M, and Wöll C, J. Am. Chem. Soc. 128, 13868 (2006).

[11] Evans S. D, Johnson S, Ringsdorf H, Williams L. M, and Wolf H, Langmuir 14, 6436 (1998).

[12] Stiller B, Karageorgiev P, Perez-Enciso E, Velez M, Vieira S, Reiche J, Knochenhauer G, Prescher $\mathrm{D}$, and Brehmer L, Surface and Interface Analysis 30, 549 (2000). 
[13] Hamelmann F, Heinzmann U, Siemeling U, Bretthauser F, and Vor der Brüggen J, Applied Surface Science 222, 1 (2003).

[14] Sortino S, Petralia S, Conoci S, and Di Bella S, Journal of Materials Chemistry 14, 811 (2004).

[15] Pace G, Ferri V, Grave C, Elbing M, von Hanisch C, Zharnikov M, Mayor M, Rampi M. A, and Samorì $\mathrm{P}$, PNAS 104, 9937 (2007).

[16] Kumar A. S, Ye T, Takami T, Yu B.-C, Flatt A. K, Tour J. M, and Weiss P. S, Nano Lett. 8, 1644 (2008).

[17] Ferri V, Elbing M, Pace G, Zharnikov M, Samorì P, Mayor M, and Rampi M, Angew. Chem. Int. Ed. 47, 3407 (2008).

[18] Qune L. F. N. A, Akiyama H, Nagahiro T, Tamada K, and Wee A. T. S, Appl. Phys. Lett. 93, 083109 (2008).

[19] Crivillers N et al., Phys. Chem. Chem. Phys. 13, 14302 (2011).

[20] Gahl C, Schmidt R, Brete D, McNellis E, Freyer W, Carley R, Reuter K, and Weinelt M, J. Am. Chem. Soc. 132, 1831 (2010).

[21] Tamada K, Akiyama H, and Wei T. X, Langmuir 18, 5239 (2002).

[22] Akiyama H, Tamada K, Nagasawa J, Abe K, and Tamaki T, J. Phys. Chem. B 107, 130 (2003).

[23] Ballav N, Terfort A, and Zharnikov M, J. Phys. Chem. C 113, 3697 (2009).

[24] Madueno R, Risnen M. T, Silien C, and Buck M, Nature 454, 618 (2008).

[25] Silien C, Risnen M. T, and Buck M, Small 6, 391 (2010).

[26] Jung U, Kuhn S, Cornelissen U, Tuczek F, Strunskus T, Zaporojtchenko V, Kubitschke J, Herges R, and Magnussen O, Langmuir 27, 5899 (2011).

[27] Wagner S, Leyssner F, Kördel C, Zarwell S, Schmidt R, Weinelt M, Rück-Braun K, Wolf M, and Tegeder P, Phys. Chem. Chem. Phys. 11, 6242 (2009).

[28] Bain C. D, Evall J, and Whitesides G. M, J. Am. Chem. Soc. 111, 7155 (1989).

[29] Venkataraman N. V, Zürcher S, Rossi A, Lee S, Naujoks N, and Spencer N. D, J. Phys. Chem. C 113, 5620 (2009).

[30] Freyer W, Brete D, Schmidt R, Gahl C, Carley R, and Weinelt M, J. Photochem. Photobiol. A Chem. 204, 102 (2009).

[31] Kowalczyk P, Appl. Surf. Sci. 253, 4036 (2007).

[32] Schmidt R, McNellis E, Freyer W, Brete D, Gießel T, Gahl C, Reuter K, and Weinelt M, Appl. Phys. A 93, 267 (2008).

[33] Feulner P, Niedermayer T, Eberle K, Schneider R, Menzel D, Baumer A, Schmich E, Shaporenko A, Tai Y, and Zharnikov M, Phys. Rev. Lett. 93, 178302 (2004).

[34] Heister K, Rong H.-T, Buck M, Zharnikov M, Grunze M, and Johansson L. S. O, J. Phys. Chem. $B$ 105, 6888 (2001).

[35] Ge Y, Weidner T, Ahn H, Whitten J. E, and Zharnikov M, J. Phys. Chem. C 113, 4575 (2009).

[36] E. McNellis and K. Reuter, unpublished.

[37] Wang L, Rangger G. M, Romaner L, Heimel G, Bučko T, Ma Z, Li Q, Shuai Z, and Zojer E, Adv. Funct. Mater. 19, 37663775 (2009).

[38] Egger D. A, Rissner F, Rangger G. M, Hofmann O. T, Wittwer L, Heimel G, and Zojer E, Phys. Chem. Chem. Phys. 12, 4291 (2010).

[39] Kuhn H and Kuhn C, in J-Aggregates, edited by Kobayashi T (World Scientific, ADDRESS, 1996), pp. 1-40.

[40] Schmidt R, Hagen S, Brete D, Carley R, Gahl C, Dokic J, Saalfrank P, Hecht S, Tegeder P, and Weinelt M, Phys. Chem. Chem. Phys. 12, 4488 (2010).

[41] Ballav N, Schüpbach B, Dethloff O, Feulner P, Terfort A, and Zharnikov M, J. Am. Chem. Soc. 129, 15416 (2007).

[42] Hallmann L, Bashir A, Strunskus T, Adelung R, Staemmler V, Wöll C, and Tuczek F, Langmuir 24, 5726 (2008).

[43] Ballav N, Schüpbach B, Neppl S, Feulner P, Terfort A, and Zharnikov M, J. Phys. Chem. C 114, 12719 (2010). 
[44] Neppl S, Bauer U, Menzel D, Feulner P, Shaporenko A, Zharnikov M, Kao P, and Allara D, Chem. Phys. Lett. 447, 227 (2007).

[45] Carniato S, Ilakovac V, Gallet J.-J, Kukk E, and Luo Y, Phys. Rev. A 71, 022511 (2005).

[46] Carniato S, Taïeb R, Kukk E, Luob Y, and Brena B, J. Chem. Phys. 123, 214301 (2005).

[47] Stöhr J, Outka D. A, Baberschke K, Arvanitis D, and Horsley J. A, Phys. Rev. B 36, 2976 (1987).

[48] Bagus P. S, Weiss K, Schertel A, Wöll C, Braun W, and C. Hellwig C. J, Chem. Phys. Lett. 248, 129 (1996).

[49] Ishii I, McLaren R, Hitchcock A. P, Jordan K. D, Choi Y, and Robin M. B, Can. J. Chem. 66, 2104 (1988).

[50] Ågren H, Carravetta V, Vahtras O, and Pettersson L. G. M, Phys. Rev. B 51, 17848 (1995). 